\title{
Analyse et maîtrise des vibrations d'une conduite forcée extérieure
}

\author{
Analysis and control of vibrations in an exterior penstock
}

\author{
par I. Turchet \\ EDF - CNEH \\ T. Cousot \\ $\mathrm{EDF}-\mathrm{CNEH}$
}

The vibration problem in exterior penstocks presented here has been resolved whereas the authors know few things about these vibrations. They calculated the eigen frequencies and penstock constraints while it was working and provided necessary stiffnings. They set up a calculation procedure which used problems resolution with fluid-structure interactions. Hypotheses were confirmed during the service trial and were validated. This has led to a better understanding of resonance phenomena due to plane waves in penstocks

\section{INTRODUCTION}

Les vibrations de conduites forcées sont des phénomènes peu fréquents. A chaque fois que ce problème se produit, il est résolu par une campagne d'essais permettant la mise en évidence des modes mis en résonance et la mesure des contraintes subies par la conduite. Dans la plupart des cas, ces contraintes sont acceptables. Dans les cas restants, un raidissement de la conduite est nécessaire.

Le problème exposé ci-après est de ce type, sa particularité venant de la pauvreté des informations sur les vibrations recueillies sur site, la conduite étant pleine.

\section{II — PRÉSENTATION DU PROBLÈME}

La conduite forcée dont il est question alimente une station de pompage turbinage comportant un groupe de 165 MW de puissance maximale sous $500 \mathrm{~m}$ de chute environ. Son diamètre est $3,5 \mathrm{~m}$ et sa longueur de $1200 \mathrm{~m}$ environ.

Lors de la mise en service, un incident conduisit à une interruption des essais de plusieurs mois. Afin de l'assister pour la mise en service de son unité dans de bonnes conditions, le maître d'ouvrage eut recours à EDF-CNEH.

En marge du problème qui avait causé l'interruption de la mise en service, les quelques essais réalisés avaient permis de constater que lors du fonctionnement en marche à vide dans le sens turbine, la partie supérieure de la conduite forcée, située à plus d'un kilomètre de la machine était sujette à des vibrations. Les seules informations concernant ces vibrations furent des mesures ponctuelles de déplacement de la coque, mais sans information ni sur leurs fréquences, ni sur leurs phases. Par ailleurs, d'autre mesures avaient mis en évidence des fluctuations de pression dont le spectre se situait entre 8 et $12 \mathrm{~Hz}$, mais avec de faibles amplitudes.

Une particularité de la conduite forcée est qu'elle longe sur la majeure partie de son parcours d'autres conduites forcées alimentant une usine voisine et surtout une piste très fréquentée menant à un village. Avec l'apparition de ces vibrations, le maître d'ouvrage eut donc à faire face à un problème imprévu de sûreté de ses installations.

Plusieurs questions se sont alors posées :

- quelle était la source des vibrations de la conduite forcée,

- ces vibrations étaient-elles nocives,

- si oui, devait-on atténuer la source ou modifier la structure ?

Après observation des installations et réflexions il fut reconnu que la turbine était responsable des vibrations de la conduite. La réduction des fluctuations de pression à la source n'étant pas possible, il restait à connaître la nocivité de ces vibrations et éventuellement à en réduire l'amplitude. Le problème n'apparaissait qu'en marche à vide, fonctionnement habituellement peu fréquent, mais qui, sur cette installation, se produit sur de plus longues périodes, ce qui ne permettait pas de négliger l'effet de fatigue de la structure.

Les quelques mois de report de la mise en service devaient être mis à profit pour faire les calculs nécessaires et apporter les modifications jugées utiles. La reprise des essais permit par la suite de vérifier par des mesures, les calculs et l'efficacité des modifications. EDF fut chargé par le maître d'ouvrage de mener ce travail. 


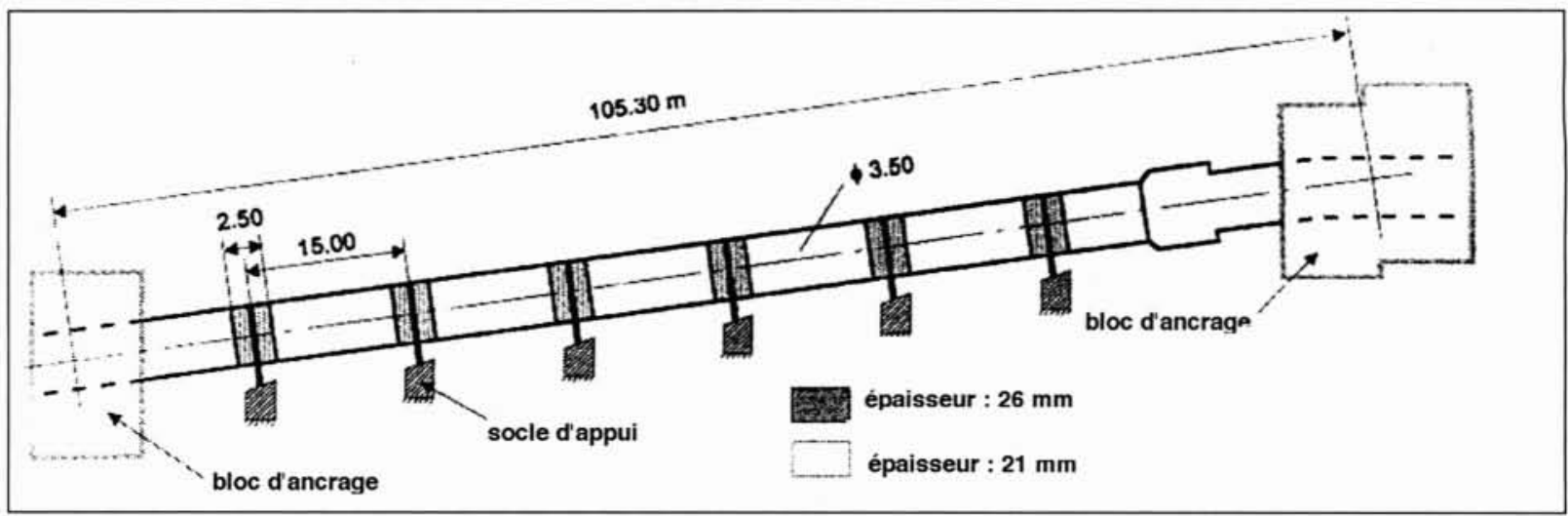

1. Tronçon de $107 \mathrm{~m}$ de la conduite étudiée.

\section{III — MODÉLISATION DE LA CONDUITE}

\section{- 3.1 Objectifs de la modélisation}

Le souhait du maître d'ouvrage était de pouvoir assurer un fonctionnement sûr de la conduite forcée y compris en phase de marche à vide. Cela n'excluait pas d'admettre des vibrations, pour autant que l'endommagement correspondant soit faible. Toutefois, pour des raisons d'ordre psychologique, le maître d'ouvrage insista pour que les fréquences de vibration soient suffisamment éloignées de la plage d'excitation de la turbine.

\subsection{Données et hypothèses}

Parmi les quatre tronçons de la conduite sujets à des vibrations, nous ne présentons les résultats que de l'un d'eux, les autres ne présentant pas de différences fondamentales.

Ce tronçon de $107 \mathrm{~m}$ est composé de 6 portées de $15 \mathrm{~m}$ et d'une de $6 \mathrm{~m}$. Les deux extrémités sont encastrées dans des blocs d'ancrage en béton, tandis que chaque portée repose sur des appuis glissants. En partie supérieure se trouve un joint de dilatation. Le diamètre intérieur de la conduite est de $3,5 \mathrm{~m}$. Les figures 1 et 2 donnent les dimensions de la conduite ainsi que quelques détails des renforts et des appuis.

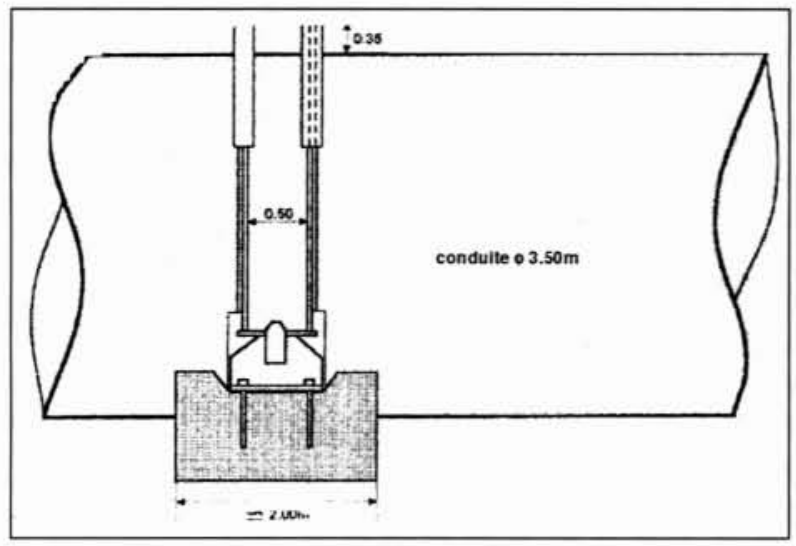

2. Détail d'un appui avec raidisseur.

Déplacements maximum mesurés lors des vibrations : $5 \mathrm{~mm}$ crête à crête.
Caractéristiques de l'acier utilisé : Module d'Young $=2,1$. $1011 \mathrm{~Pa}$, Coefficient de Poisson $=0,3$, Masse volumique $=$ $7850 \mathrm{~kg} / \mathrm{m}^{3}$.

Les critères d'acceptabilité des vibrations étaient :

- Contraintes inférieures à la limite de fatigue de $26 \mathrm{MPa}$ crête à crête selon $\mathrm{CECM} \mathrm{n}^{\circ} 43$,

- Fréquences de vibrations des modes excitables en dehors de la plage de $[8 ; 12] \mathrm{Hz}$ et si possible supérieures à $12 \mathrm{~Hz}$.

\section{- 3.3 Procédure}

\subsection{Etape I : détermination du type de mode en jeu}

A priori, deux types de mode peuvent être excités par des fluctuations de pression : des modes de flexion en poutre où l'on considère que la section de la conduite garde sa forme initiale, les modes de type coque où la section se déforme et éventuellement des modes couplés, combinaison des deux précédents.

Les fluctuations de pression se manifestent par des ondes planes ou sphériques. Cependant ce sont les ondes planes qui se propagent le plus facilement à partir de leur source. Les ondes sphériques, elles, se transforment généralement en ondes planes quelques diamètres hydrauliques après leur source. En raison des distances mises en jeu dans notre cas, il est certain que nous n'étions en présence que d'ondes planes.

Les mécanismes permettant d'exciter une tuyauterie de type industrielle à partir d'ondes planes sont bien connus et se modélisent aisément. La transmission des vibrations du fluide à la coque et vice-versa se fait par les coudes et les changements de section. Les modes en jeu sont de type flexion en poutre.

Dans le cas des conduites forcées, ce type de mécanisme ne se retrouve quasiment jamais car les coudes sont généralement bloqués dans des blocs d'ancrage lourds et rigides, comme c'est le cas pour notre conduite.

Pour expliquer les mécanismes d'excitation, il faut tenir compte des déformations sous poids propre des conduites. Le chapitre 3 traite de ce mécanisme dans le cas des modes de coque.

Pour savoir de quel type de mode il était question sur notre conduite, nous avons fait quelques calculs préliminaires en poutre. Ces calculs qui ne présentent que peu d'intérêt, ont montré que les fréquences des modes de poutre étaient situées en dehors de la plage de fréquences à éviter. 


\subsubsection{Etape 2 : calcul des fréquences propres de la conduite en eau}

La mise au point du modèle d'une structure existante nécessite le recalage de certains paramètres sur des données physiques. Or aucune mesure de fréquence n'avait pu être réalisée sur la conduite pleine d'eau. C'est donc sur la conduite vide qu'EDF - DTG a réalisé une analyse modale afin d'obtenir les informations nécessaires au recalage. Le recalage a porté essentiellement sur la raideur des raidisseurs dont la forme est complexe. Ceux-ci ont simplement été modélisés par des surépaisseurs localisées de la coque dont il s'agissait de déterminer les valeurs convenables.

Par ailleurs, pour tenir compte de l'effet de masse ajoutée de l'eau, deux méthodes sont possibles : soit un modèle intégrant des éléments de volume fluide avec interaction fluidestructure, soit un modèle " vide " pour lequel la masse volumique de l'acier tient compte de la masse ajoutée de l'eau. Du fait de difficultés de mise au point des modèles fluide-structure et de temps de calcul, c'est la seconde solution que nous avons retenue. Toutefois, nous avons dû au préalable calculer les pourcentages de masse ajoutée à utiliser, sachant que ceux-ci sont dépendants du type de mode. Pour cela nous avons constitué un modèle fluide-structure de taille réduite composé d'une portée de conduite forcée (portion comprise entre deux appuis simples) dont nous avons calculé les fréquences propres des deux premiers modes (2 et 3 lobes). Puis par tâtonnement, nous avons cherché la masse volumique de l'acier à prendre en compte sur un modèle ne comportant que la coque de la conduite (modèle vide), et ce pour chacun des deux modes.

Cette partie s'est donc déroulée de la façon suivante :

- constitution des modèles des différents tronçons, calcul des fréquences propres,

- analyse modale de la conduite vide.

- recalage du modèle en air,

- recherche des pourcentages de masse ajoutée à l'aide d'un modèle fluide structure composé d'une portée de conduite forcée et du même modèle "vide",

- calculs des fréquences propres de la structure complète en eau en appliquant les masses ajoutées déterminées à l'étape précédente et donc obtention des modes susceptibles d'être excités par les fluctuations de pression.

\subsubsection{Etape $3:$ déterminer les contraintes subies par la structure}

Cette étape consistait à calculer les contraintes subies par la structure lors des épisodes de vibrations constatés avant l'arrêt de la mise en service. Les seules informations disponibles étaient des mesures de déplacement selon deux génératrices en quelques points. Pour en déduire les contraintes, nous avons effectué une appropriation modale. La structure modélisée a été excitée par une force dont les caractéristiques étaient les suivantes :

- amplitude arbitraire,

- fréquence voisine de celle du mode le plus proche de la fréquence des fluctuations de pression,

- localisation sur un ventre du mode.

Cette opération a été réalisée pour tous les modes susceptibles d'être excités.

\subsubsection{Etape 4 : définition des modifications nécessaires.}

Les résultats obtenus aux étapes 2 et 3 ont été comparés aux critères présentés ci-dessus. Nous avons été conduits à proposer des modifications de la conduite consistant simplement à ajouter des raidisseurs supplémentaires. Les raidisseurs étaient du même type que ceux déjà en place, et leur espacement fut déterminé à l'aide de calculs de fréquence propre. Aucun calcul de contrainte par appropriation modale n'a évidemment été fait sur la structure modifiée par manque de données sur les forces d'excitation réelles.

\subsection{Calculs et Résultats}

\subsection{Modélisation}

Les calculs du tronçon ont été faits à l'aide du code de mécanique ASTER développé par EDF.

L'ensemble du tronçon a été modélisé à l'aide d'éléments de coque majoritairement à 4 nœuds mesurant environ $0,5 \mathrm{~m}$ de côté. La circonférence de la conduite a été découpée en 24 éléments, soit au total plus de 5000 éléments.

Les extrémités de la conduite étaient bloquées, tandis que les raidisseurs liés à des supports étaient libres en translation selon l'axe de la conduite. L'effet de raidissement de la pression n'a pas été pris en compte, ce qui nous a donné un caractère conservatif aux résultats.

Le modèle fluide structure d'un tronçon de $15 \mathrm{~m}$ a été réalisé selon les mêmes caractéristiques que ci-dessus, en y ajoutant des éléments de type fluide. Les calculs ont été menés à l'aide du code ANSYS 5.2

\subsubsection{Recalage}

Le recalage s'est fait à partir de l'analyse modale en air dont il a été extrait 2 modes de coque à deux lobes se différentiant par une répartition différente des lobes longitudinaux. Les fréquences de ces modes étaient de 17,6 et 19,4 Hz. Ces deux modes ont été retrouvés par le calcul. L'épaisseur de coque nécessaire pour simuler les raidisseurs était de $200 \mathrm{~mm}$ (soit $3 \mathrm{dm}^{4}$ par raidisseur, valeur légèrement supérieure à l'inertie des Tés formant les raidisseurs). Les fréquences obtenues alors sur le modèle mathématique en air ont été respectivement de 17,7 et $19,1 \mathrm{~Hz}$, valeurs suffisamment proches des valeurs expérimentales.

\subsubsection{Résultats}

Les calculs permirent de mettre en évidence deux modes aux déformées très régulières comme le montrent les figures ci-dessous. Le premier de fréquence $8,6 \mathrm{~Hz}$ est un mode à 2 lobes circonférentiels et le second à $10,1 \mathrm{~Hz}$ est un mode à 3 lobes circonférentiels. Chaque portée se comporte de manière identique.

Ces résultats sont donc venus confirmer le fait que certains modes de coque avaient leurs fréquences dans la zone de perturbation de la machine et donc étaient susceptibles d'être excités.

L'appropriation modale de chacun de deux modes permit de déterminer les contraintes de Von-Mises subies lors des vibrations. Ainsi on a pu obtenir les valeurs suivantes :

— mode à 2 lobes : 8,6 Hz ; 9,5 MPa (Figure 3) mode à 3 lobes : $10,1 \mathrm{~Hz} ; 8,11 \mathrm{MPa}$ (Figure 4).

Les maxima de contrainte se localisent à proximité des renforts, la contrainte principale maximale étant orientée suivant l'axe de la conduite. 


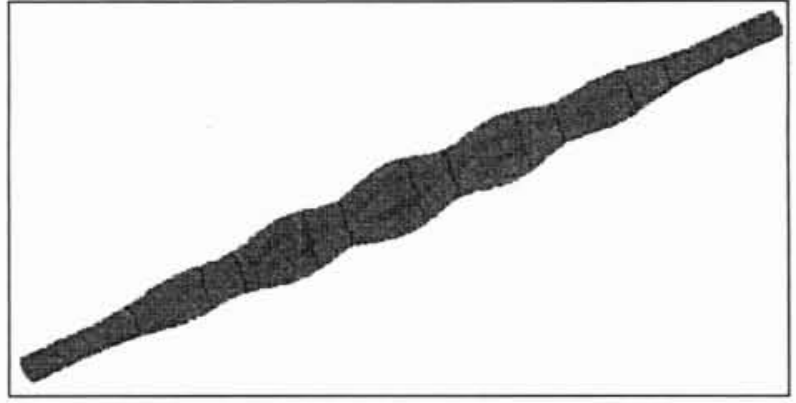

3. Déformation (exagérée) du mode à 2 lobes.

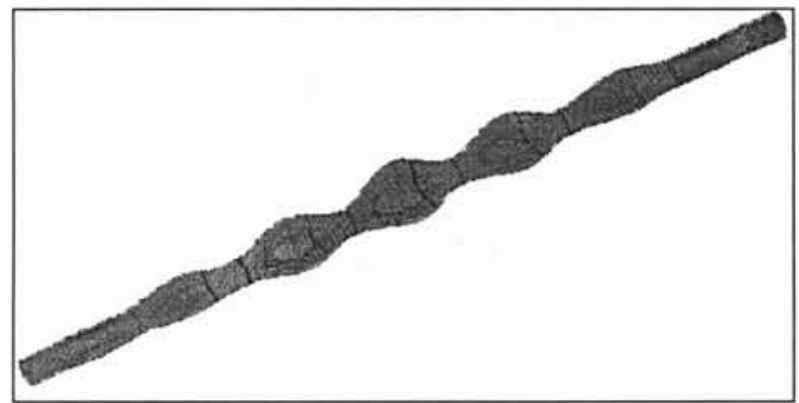

4. Déformation (exagérée) du mode à 3 lobes.

Bien que les contraintes soient faibles, les fréquences des deux modes sont situées dans l'intervalle à éviter et nous avons donc proposé un raidissement de la conduite. Pour cela, des raidisseurs de même type que ceux existants ont été ajoutés au modèle de telle sorte que l'on ait un raidisseur tous les 5 mètres. Le nombre de raidisseurs est donc passé de 1 à 3 par tronçon. Les calculs fournirent les résultats suivants :

- premier mode à 2 lobes, 17,2 Hz (Figure 5)

- premier mode à 3 lobes, $>20,9 \mathrm{~Hz}$

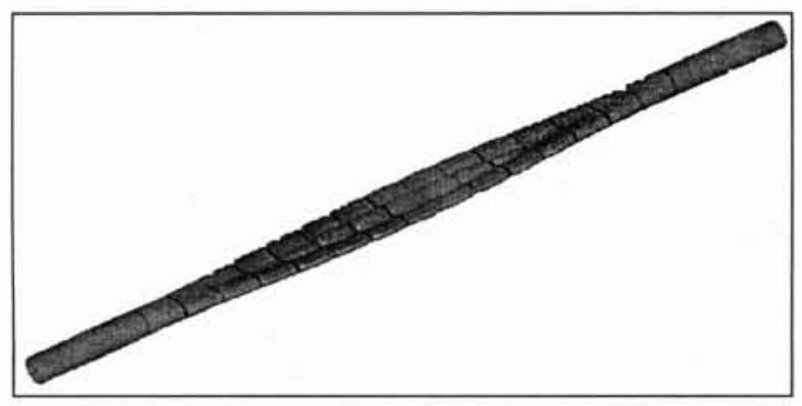

5. Déformation (exagérée) du mode à 2 lobes de la conduite raidie.

La déformée du mode à 2 lobes qui est présentée en figure 5 est très différente des résultats précédents. Ici la conduite se comporte pratiquement comme une seule portée de 107 $\mathrm{m}$. Il est prévisible que ce genre de mode par rapport aux modes précédents, est certainement beaucoup plus difficile à mettre en résonance et que les mécanismes conduisant à son apparition sont de nature différente.

On note également le fait que notre calcul n'intégrait pas le raidissement dû à la pression ; les fréquences réelles sont en effet légèrement supérieures à ce qui a été calculé. Ainsi les résultats sont entachés d'une erreur allant dans le sens de la sécurité.

\subsection{Confrontation aux résultats d'essais}

Les travaux étant achevés, la reprise de la mise en service fut l'occasion de contrôler le comportement de la conduite pendant les phases de marche à vide et de vérifier certaines de nos hypothèses.

Les mesures réalisées permirent de confirmer que la conduite était bien excitée sur des modes propres, le contenu spectral se limitant à une fréquence dominante accompagnés parfois de quelques autres pics de moindre importance. D'autre part les modes mis en résonance étaient bien des modes de coque.

Malheureusement, nous n'avons pas pu comparer les fréquences calculées et mesurées de la structure modifiée du fait de la non-conformité des modifications apportées par rapport à ce qui avait été demandé. En effet, les raidisseurs mis en place avaient une raideur très inférieure à celle qui était prévue. Toutefois nous avons pu constater que les vibrations du tronçon avaient une fréquence supérieure à $12 \mathrm{~Hz}$ (autour de $13,14 \mathrm{~Hz}$ ) et que donc les raies principales des fluctuations de pression issues de la machine, entre 8 et $12 \mathrm{~Hz}$, ne l'affectaient plus.

\section{IV — ONDES PLANES ET MODES DE COQUE}

Comme il a été expliqué plus haut, on peut supposer que ce sont les défauts de forme qui permettent d'expliquer l'interaction entre le fluide et la structure. En effet dans un tuyau parfaitement rectiligne et de section circulaire, les ondes planes ne peuvent provoquer qu'un gonflement de la coque qui reste circulaire.

En revanche un faux rond et donc la perte de l'axisymétrie de la section et la présence d'une éventuelle flexion en poutre permettront l'apparition de modes de coque, alors que l'excitation est, elle, axisymétrique et uniforme. C'est ce que nous allons mettre en évidence dans les calculs numériques suivants.

\subsection{Procédures}

Nous avons effectué ce calcul en nous basant sur une portée de même type que celle de la conduite forcée précédemment étudiée. Seule une demi-longueur de portée a été modélisée avec application des conditions de symétrie aux deux extrémités, si bien que le modèle est équivalent à une conduite de longueur infinie simplement supportée à intervalles réguliers. Les renforts ont été pris identiques à ceux de la conduite réelle. Les épaisseurs retenues sont légèrement différentes, avec $24 \mathrm{~mm}$ pour la partie courante et 29 $\mathrm{mm}$ pour les parties raidies.

Le code utilisé ici est SYSTUS+ 1.1.

Le calcul consistait à mettre en évidence la mise en résonance d'un tuyau selon un mode de coque, par une fluctuation de pression interne. Le tuyau initialement rectiligne et circulaire est d'abord soumis aux efforts statiques du poids et de la pression qui représentent donc une précontrainte. Nous avons procédé ainsi :

1. Calculs statiques. Application du poids (tuyau plein d'eau) et de la pression,

2. Calcul de la matrice de raideur de la structure précontrainte par la déformation statique,

3. Application à la structure du pourcentage de masse ajoutée correspondant au mode à 2 lobes et analyse modale avec la matrice de rigidité précontrainte, 
4. Analyse harmonique avec matrice de rigidité précontrainte et masse ajoutée. Application d'une pression interne fluctuante à une fréquence très proche de la fréquence du mode propre à 2 lobes circonférentiels et 1 lobe longitudinal.

On constate que les calculs modaux et harmoniques n'utilisent pas les possibilités de l'interaction fluide-structure du code. En effet, l'utilisation d'une structure précontrainte n'était pas compatible avec un calcul de ce type.

\subsection{Résultats}

\subsection{Calcul statique}

Le poids propre et la pression induisent sur le tuyau des déformations de plusieurs types. La pression conduit à augmenter le rayon de façon uniforme de $\Delta \mathrm{R}=P R^{2} / E e$, où $\mathrm{P}, \mathrm{R}$, $E$ et e sont respectivement la pression, le rayon, le module d'Young et l'épaisseur. Ici, nous avons une augmentation de rayon de $1,1 \mathrm{~mm}$ environ. Le poids propre a deux effets : il provoque un fléchissement de la conduite (de l'ordre de 1,1 $\mathrm{mm}$ au milieu), et une déformation de la section du tuyau. Pour le tuyau décrit ci-dessus et une pression de 20 bars, la déformation radiale de la section dont est responsable le poids est représentée aux figures 6 et 7 , déduction faite de la flèche de l'ensemble du tuyau et du gonflement du à la pression.

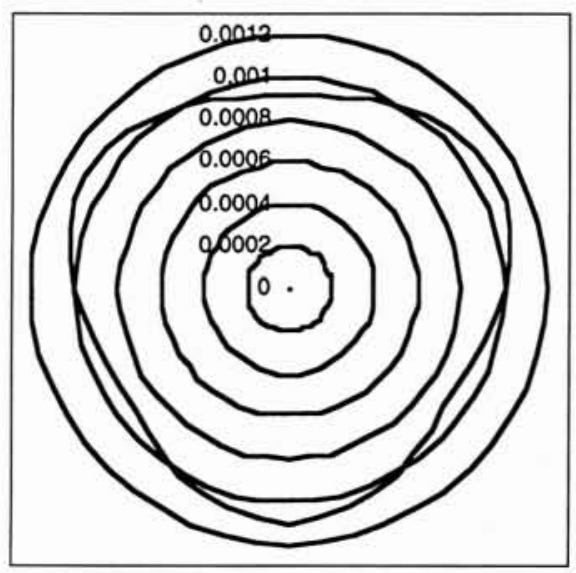

6. Déformation exagérée de la section sous l'effet du poids propre (coque+eau).

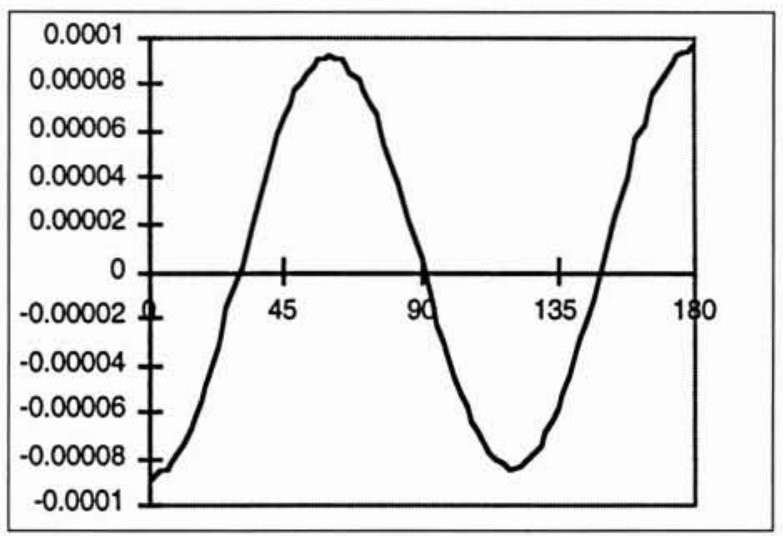

7. Déplacement radial du tuyau (en mètres) sous l'effet du poids propre (coque+eau).

Cette déformation est faible, atteignant $0,09 \mathrm{~mm}$. Rapportées respectivement à la longueur et au rayon, la flèche et le faux-rond ont une valeur relative de $7 \cdot 10^{-5}$ et $5.10^{-5}$. On peut affirmer que ces valeurs sont inférieures aux défauts de forme dus à la construction des viroles. Cependant elles seront suffisantes pour simuler l'excitation des modes de coque.

\subsubsection{Analyse modale}

L'analyse modale de la structure avec masse ajoutée et pression interne conduit à une fréquence de $12,03 \mathrm{~Hz}$ pour le premier mode de coque à 2 lobes circonférentiels et un lobe longitudinal.

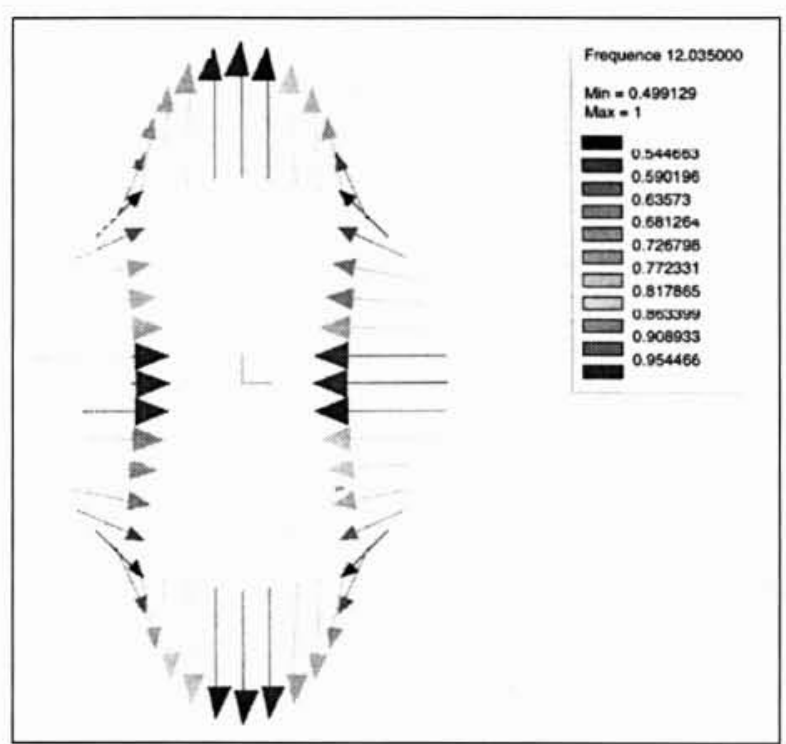

8. Déplacements absolus de la section médiane du tuyau pour le ler mode à 2 lobes.

Un calcul similaire a été fait en appliquant seulement la précontrainte due à la pression. Nous avons pu noter que les quatre premiers modes étaient quasiment identiques en fréquence et en déformée dans les deux cas de précontrainte. C'est seulement à partir du cinquième mode que les deux modèles commencent à se différencier. Cette constatation est certainement due au fait que nous sommes en présence d'un défaut de très faible amplitude.

\subsubsection{Calcul harmonique}

Le calcul harmonique a consisté à exciter la structure avec une pression fluctuante d'amplitude 2 bars et de fréquence $12,03 \mathrm{~Hz}$ identique à celle du premier mode de coque. La pression était uniformément répartie sur la surface interne du tuyau.

Deux calculs ont été réalisés, l'un sur la structure précontrainte par la pression seule et l'autre sur la structure précontrainte par la pression et le poids propre.

La Figure 9 présente le premier calcul. Le tuyau est initialement circulaire et le reste sous l'effet de l'excitation. Les amplitudes indiquées sont dues à la combinaison de la pression statique $(0,00112 \mathrm{~m})$ et de la fluctuation de pression. On voit que cette dernière amène des amplitudes négligeables ; cela indique que ce type d'excitation n'est pas apte à faire vibrer la conduite selon le mode de coque.

La Figure 10 correspondant au deuxième calcul fait apparaître la combinaison de deux déformations. La première est une ovalisation selon le premier mode de coque décrit plus haut. La deuxième est un déplacement vertical qui indique que le mode de coque est alors couplé à un mode de flexion d'ensemble du tuyau, de type poutre.

\subsubsection{Conclusion}

Les deux derniers calculs montrent que c'est par la pré- 


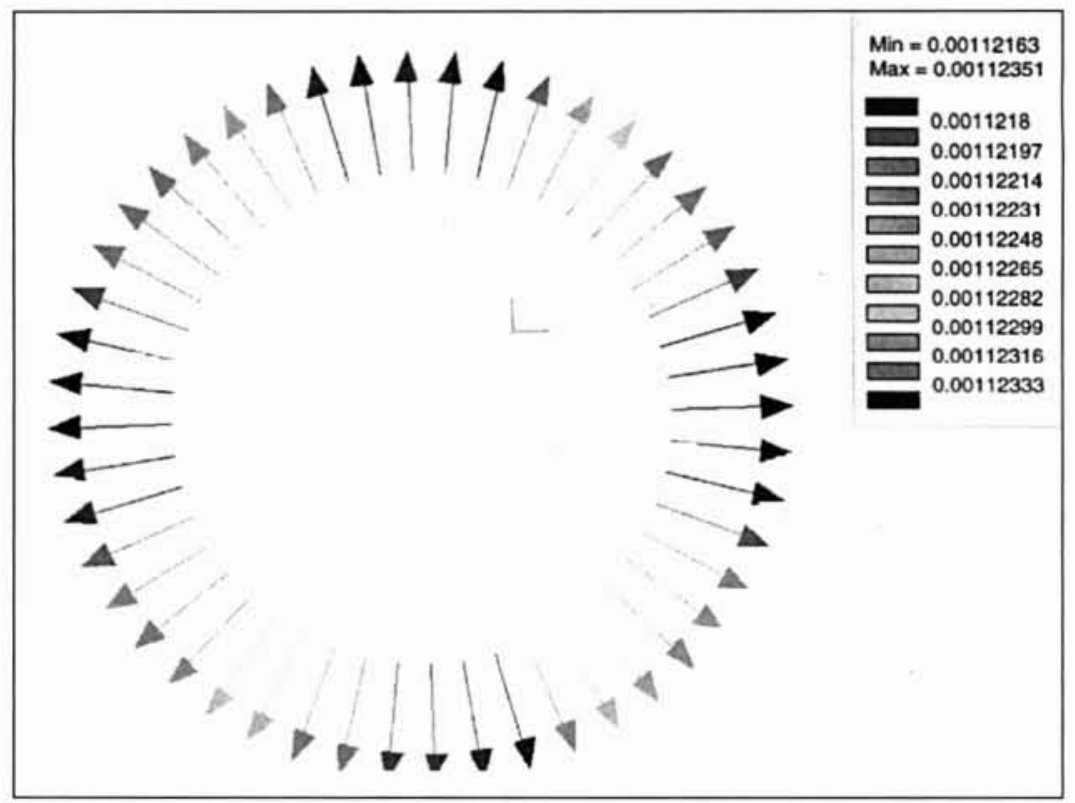

9. Déplacements absolus de la section médiane sous l'effet des pressions statique et fluctuante - conduite initialement circulaire. (La fréquence indiquée est en fait une pulsation).

10. Déplacements absolus de la section médiane sous l'effet des pressions statique et fluctuante conduite initialement déformée sous l'effet du poids propre et de la pression. (La fréquence indiquée est en fait une pulsation).

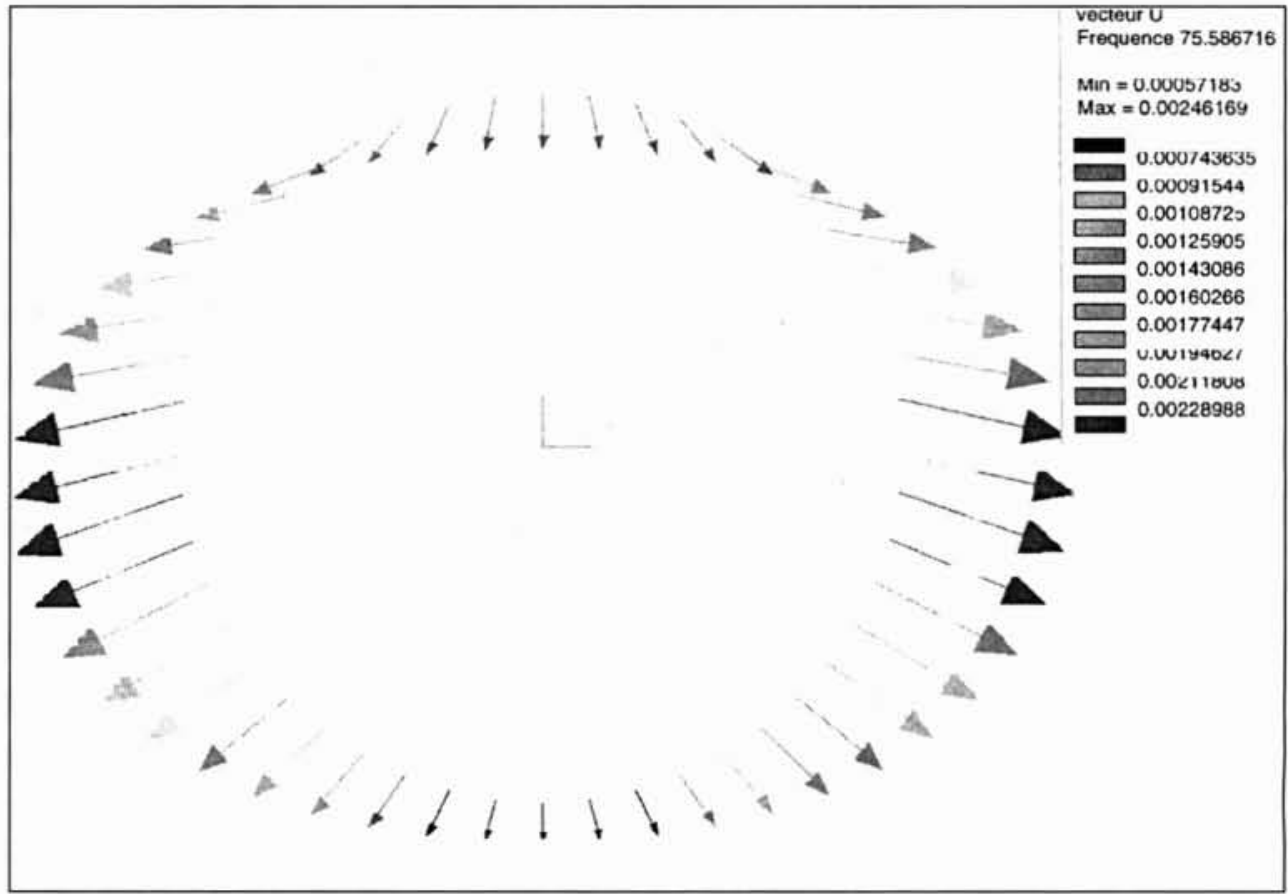

sence d'un défaut de forme qu'une excitation d'amplitude uniforme sur la circonférence est capable de mettre une conduite en résonance selon un mode de coque. D'autre part, la fréquence des premiers modes n'est pas modifiée par le défaut de forme qui est dans notre cas de faible amplitude.

\section{V —SYNTHÈSE}

Le problème de vibrations de conduite forcée présenté ici a la particularité d'avoir été résolu alors que nous ne disposions que de très peu d'informations sur ces vibrations. Le problème posé consistait à calculer les fréquences propres et les contraintes de la conduite en fonctionnement et à prévoir les raidissements nécessaires. Cela a été réalisé par la mise en place d'une procédure de calcul utilisant en particulier la résolution de problèmes avec interaction fluide-structure, grâce à une analyse modale de la conduite vide qui a permis un recalage de certains paramètres mécaniques et enfin grâce à certaines hypothèses sur le type de modes en jeu.

Les hypothèses utilisées ont été confirmées lors des essais de mise en service de l'installation et ont ensuite été validées de façon numérique. Il a été mis en évidence que les ondes planes sont susceptibles d'engendrer des modes de coques à condition d'être en présence de défauts de forme du tuyau. 